\title{
High-resolution DOA estimation with Meridian prior
}

\author{
Guanghui Zhao ${ }^{1 *}$, Jie Lin ${ }^{1}$, Fangfang Shen ${ }^{1}$, Guangming Shi ${ }^{1}$, Qingyu Hou ${ }^{2}$ and Zicheng Liu ${ }^{1}$
}

\begin{abstract}
Based on the assumption that only a few point sources exist in the spatial spectrum, the direction-of-arrival (DOA) estimation problem can be formulated as a problem of sparse representation of signal with respect to a dictionary. By choosing a proper dictionary, the array measurements can be well approximated by a linear combination of a few entries of the dictionary, in which the non-zero elements of the sparse coefficient vector correspond to the targets' arrival direction. Conventionally, the desired sparsity of signal is guaranteed by imposing a constraint of Laplace prior on the distribution of signal. However, its performance is not satisfied under the condition of insufficient data or noisy environment since a lot of false targets will appear. Considering that the Meridian distribution has the characteristic of high energy concentration, we propose to adopt the Meridian prior as the prior distribution of the coefficient vector. Further, we present a new minimization problem with the Meridian prior assumption (MMP) for DOA estimation. Because the Meridian prior imposes a more stringent constraint on the energy localization than the Laplace prior, the proposed MMP method can achieve a better DOA estimation, which is embodied in higher resolution and less false targets. The experiments of both simulation and ground truth data process exhibit the superior performance of our proposed algorithm.
\end{abstract}

Keywords: Direction-of-arrival (DOA); Sparse representation; High resolution; Laplace prior; Meridian prior

\section{Introduction}

In many fields, including radar, sonar, and medical signal processing, one of the most highly explored research problems is how to determine the precise direction-of-arrival (DOA) of multiple incident signals from noisy measurements of a sensor array. Among the various methods for solving the DOA problem, such as multiple signal classification [1], estimation of signal parameters via rotational invariance techniques [2], and beamforming (BF) [3], the BF method is a popular one. However, it is unable to separate the closely spaced sources, when the angular interval is smaller than the Rayleigh resolution limit. This resolution limitation can be overcome by some high-resolution DOA estimation algorithms [4-6], in which a representative class is the subspace-based method. Unfortunately, the requirement of sampling snapshot affects the DOA estimation performance, especially in the noisy environment.

The sparse representation, which has evolved rapidly in the past several years, provides a novel methodology for the DOA problem. A sparse signal is also known as a localized energy signal [7], in which zero-value entries will be

\footnotetext{
* Correspondence: ghzhao@xidian.edu.cn

'School of Electronic Engineering, Xidian University, Xi'an 710071, China Full list of author information is available at the end of the article
}

everywhere except on a minimal support of the signal space. The concept of spatial sparsity for DOA estimation was first introduced in [8], where the DOA estimation problem is formulated as a dictionary selection problem after the spatial area of interest is divided into an equalspaced angle grid. Assume that only a few point sources exist in the spatial spectrum. By choosing a proper dictionary, the array measurements can be well approximated by a linear combination of a few entries of the dictionary, in which the representation coefficient vector of the array measurements will be sparse. Then, the classical DOA problem can be formulated as a sparse representation problem, in which the sparsity constraint on the coefficient vector is manifested as a minimization of $l_{1}$-norm in the objective function.

However, the popular constraint of minimizing $l_{1}$-norm does not fully exploit the sparsity of the coefficient vector, especially under the condition that the number of point sources increases or/and the number of array measurements decreases. In [7,9], Rao and Gorodnitsky indicated that minimizing the $l_{0<p<1}$-norm of the object vector which is sparse can obtain a more precise result than minimizing its $l_{1}$ norm, because the $l_{0<p<1}$-norm of a vector can characterize the feature of sparsity better. Under the same assumption, a recent work of Hyder [10] shows that a higher angular

\section{实}

(c) 2013 Zhao et al.; licensee Springer. This is an Open Access article distributed under the terms of the Creative Commons Attribution License (http://creativecommons.org/licenses/by/2.0), which permits unrestricted use, distribution, and reproduction in any medium, provided the original work is properly cited. 
resolution can be achieved when an enhanced constraint is imposed on the minimal support of the energy signal, even though the amount of samples considerably reduces.

Inspired by the previous work, in this paper, we impose a Meridian prior [11] as the sparsity constraint for the spatial spectrum and then develop a new minimization problem with the Meridian prior assumption (MMP) to achieve high-resolution DOA estimation for the point source scene. Since the Meridian prior distribution can impose a more stringent constraint on the sparsity property compared with the classical $l_{1}$-norm model, a more accurate spectrum for DOA estimation can be obtained even when only one snapshot is available. Experimental results show that the proposed algorithm is with good robustness even when the array data are insufficient.

The remainder of this paper is organized as follows. In Section 2, the transform from a DOA estimation problem to a sparse representation problem is briefly presented. In Section 3, Meridian distribution is analyzed, and a new optimization function with Meridian prior assumption is proposed. The detailed algorithm for high-resolution DOA estimation is discussed in Section 4. Numerical simulations presented in Section 5 confirm the superior performance of the proposed algorithm. Finally, some conclusions are drawn in Section 6.

\section{Sparse representation-based DOA formulation}

\subsection{Problem formulation}

Consider a uniform linear phase array (ULPA) that is composed of $L$ isotropous sensors. Assume that $P$ narrowband sources from the far field impinge on the ULPA from $P$ distinct directions $\theta=\left[\theta_{1}, \theta_{2}, \ldots, \theta_{P}\right]$ with plane waves. The first element in the array is taken as the phase reference. Then, the response of the antenna array to the $i$ th impinging plane waves, also known as steering vector, can be denoted by $a\left(\theta_{i}\right)=\left(e^{-j \omega_{c} \tau_{1}\left(\theta_{i}\right)}, e^{-j \omega_{c} \tau_{2}\left(\theta_{i}\right)}, \ldots, e^{-j \omega_{c} \tau_{L}\left(\theta_{i}\right)}\right)$, in which $\omega_{c}$ and $\tau_{p}$ represent the angular frequency and echo delay, respectively. After the substitution of $\omega_{c}=2 \pi f$ and $\tau_{p}\left(\theta_{i}\right)=$ $(p-1) d \cos \left(\theta_{i}\right) / c$, where $d$ is the inter-sensor spacing which is set to be $d=\lambda / 2, \lambda=c / f$ is the wavelength, and $c$ is the speed of the propagation, we can reform the vector to be $a$ $\left(\theta_{i}\right)=\left(1, e^{-j \pi \cos \left(\theta_{i}\right)}, \ldots, e^{-j \pi \cos \left(\theta_{i}\right)(L-1)}\right)$. Therefore, the output of the array can be expressed as follows:

$$
y=A s+n,
$$

where $s \in \mathbb{C}^{P \times 1}$ is the signal amplitude, $y \in \mathbb{C}^{L \times 1}$ is the observation vector, $n$ represents the additive complex noise, and the array manifold matrix $A(\theta)$ is formed with the array responses for the $P$ plane waves, $A(\theta)=\left[a\left(\theta_{1}\right), \ldots, a\left(\theta_{P}\right)\right]$. The goal of the DOA estimation discussed in this paper is to precisely locate the sources $\theta_{p}(p=1,2, \ldots, P)$, when the observation data $y$ and the steering vector $A(\theta)$ are given.

\subsection{DOA estimation as a sparse representation problem}

Since the problem of DOA estimation can be cast as a problem of sparse representation $[8,10,12]$, we discuss the representation of observation $y$ with an over-complete dictionary in this subsection. Conventionally, the spatial area of interest is partitioned into an equal-spaced angle grid. Based on this grid partition of an angle, we enumerate a finite set of possible source locations $\Theta=\left\{\vartheta_{1}, \ldots, \vartheta_{Q}\right\}$ and further form an over-complete angle dictionary $\Omega=\left\{a\left(\vartheta_{1}\right), \ldots, a\left(\vartheta_{Q}\right)\right\}$ in which the form $a\left(\vartheta_{j}\right)$ is similar to that of the steering vectors. How fine the gird is partitioned determines the number of possible locations $Q$, i.e., the number of dictionary entries. So, in order to get a high resolution, the angle grid should be partitioned as fine as possible, and the number of possible locations $Q$ should satisfy $Q>>P$. From the above procedures of generating the angle dictionary, we can conclude that, the angle dictionary $\Omega$ does not depend on the actual location of sources, so it can be constructed in advance.

We use a vector $x \in \mathbb{C}^{Q \times 1}$ to represent the target angles, and each entry of $x$ corresponds to one possible position in $\Theta$. Since $P<<Q$, vector $x$ has a sparse structure, where the $k$ th component $x_{k}$ is non-zero only if there is a target angle in this direction, i.e., $\vartheta_{k}=\theta_{l}$ for some $l$, and in this case $x_{k}=s_{l}$. Then, the DOA problem can be stated as finding a sparse vector $x$ with $P$ non-zero entries, which is an inverse problem of the following observation equations:

$$
y=\Omega x+n
$$

Since the key assumptions in (2) are the sources which can be viewed as point sources and their amount is small, this inverse problem can be settled by solving the following problem $[13,14]$ :

$$
\min _{x \in \Theta}\|y-\Omega x\|_{2}^{2}+\lambda\|x\|_{0},
$$

where $\|x\|_{0}:=\#\left\{j: x_{j} \neq 0\right\}$ is the $l_{0}$-norm of vector $x$, which counts the number of non-zero terms in $x$. As is known to all, $l_{0}$-norm characterizes the sparsity of a signal exactly, but it is hard to solve the problem with form of (3).

Candès [13] and Donoho [14] show that the optimization problem in (3) can be relaxed to a convex problem, also known the problem of basis pursuit denoising (BPDN) [15], i.e.,

$$
\min _{x \in \Theta}\|y-\Omega x\|_{2}^{2}+\lambda\|x\|_{1}
$$

where $\|x\|_{1}=\sum_{i=1}^{Q}\left|x_{i}\right|$ denotes the $l_{1}$-norm of vector $x$. The parameter $\lambda$ controls the trade-off between residual energy $\|y-\Omega x\|_{2}^{2}$ and the sparsity level of vector $x$. Since the optimization problem in (4) is convex and it can be recast as a linear programming, many algorithms (e.g., least absolute shrinkage and selection operator [16] and gradient projection for sparse reconstruction [17]) have been proposed to solve this type of optimization problem. 


\section{Robust DOA estimation with Meridian prior}

Even though solving the problem of sparse representation in (4) can obtain an improved resolution of the DOA result, the term of $l_{1}$-norm constraint does not fully exploit the sparsity of signal [18], when the array data are not only insufficient, but also heavily corrupted by the background noise. The incapability of the $l_{1}$-norm constraint in this situation usually has two consequences to the reconstructed DOA spectrum: one is missing some true targets when the targets are very close, and the other is generating some wrong targets due to the noise [19].

To overcome this disadvantage, much work has been devoted to finding a replacement of the $l_{1}$-norm constraint and developing a better approximation of the ideal sparsity constraint (the $l_{0}$-norm constraint). Specially, from the view of a Bayesian posterior estimate, Babacan [20] indicated that the minimization problem in (4) can be seen as a maximum a posteriori (MAP) criterion for estimating vector $x$, and the prior knowledge of $x$ is a Laplace distribution. Further, Rao [7] and Hyder [10] showed that if the probability density function (pdf) $f(x)$ of a distribution, as a prior of the vector $x$, falls sharply with the increase of the absolute value of variable $\left|x_{i}\right|$, the distribution can enhance the sparsity of the reconstructed vector $x$, and the higher the kurtosis of pdf is, the sparser the reconstructed signal becomes.

On the basis of the previous work, firstly, we can easily derive the MAP estimation of $x$ :

$$
\begin{aligned}
\hat{x}_{M A P}= & \underset{x}{\arg \min } \ln f(x \mid y)=\underset{x}{\arg \min }[\ln f(y \mid x)+\ln f(x)] \\
& =\underset{x}{\arg \min }\left[\ln f_{n}(y-\Omega x)+\ln f(x)\right]
\end{aligned}
$$

In order to guarantee the sparsity constraint of the signal $x$, we propose adopting Meridian distribution [11], which can offer a stronger constraint on the sparsity of the objective vector, as the prior distribution of the signal:

$$
\left\{\begin{array}{l}
f_{\text {Meridian }}\left(x_{i}\right)=\gamma\left(1+\left|\frac{x_{i}}{b}\right|\right)^{-2} \\
f_{\text {Meridian }}(x)=\gamma^{Q} \prod_{i=1}^{Q}\left(1+\left|\frac{x_{i}}{b}\right|\right)^{-2},
\end{array}\right.
$$

where $b$ denotes the signal variance, and the parameter $\gamma=\Gamma(2) / 2 b(\Gamma(1))^{2}$. Assume that the noise $n$ is Gaussian independent and identically distributed (i.i.d.) with zero mean and a variance of $\sigma_{n}^{2}$. Thus, we can obtain a new minimization problem with the Meridian prior assumption, abbreviated as MMP,
To show the superiority of the reconstruction model with Meridian prior in (6) over that with Laplace prior, the pdfs of both prior distributions with the same variance are shown in Figure 1.

Based on distribution of the signal entries in Figure 1, we can find that compared with the Laplace distribution, the rapid attenuation of the pdf function of the Meridian distribution will guarantee more signal entries which would be zero or close to zero. That is to say, the relatively small ratio of signal entries will have the most energy. Thus, for Laplace distribution as a prior of signal, the high dispersiveness of energy will result in some undesired false targets. However, for the Meridian distribution as a prior, the energy localized in a small number of entries will make the resulting signal sparser and then the reconstruction error smaller.

\section{MMP algorithm for DOA estimation}

\subsection{Algorithm formulation}

Because the objective function in (7) is non-convex, following the procedure of the focal underdetermined system solver (FOCUSS) [7,9] or iteratively reweighted least squares approach [21], we differentiate the cost function $J(x)$ as follows:

$$
\begin{aligned}
& \frac{\partial J(x)}{\partial \hat{x}}=\frac{2 \partial}{\partial \hat{x}}\left[\sum_{i=1}^{Q} \ln \left(1+\left|\frac{x_{i}}{b}\right|\right)\right]-\beta \Omega^{H}(y-\Omega \hat{x}), \\
& \frac{\partial}{\partial \hat{x}_{i}}\left[\sum_{i=1}^{Q} \ln \left(1+\left|\frac{\hat{x}_{i}}{b}\right|\right)\right]=\frac{1}{b}\left(1+\left|\frac{\hat{x}_{i}}{b}\right|\right)^{-1} \frac{\partial\left|\hat{x}_{i}\right|}{\partial \hat{x}_{i}}=\frac{1}{b}\left(1+\left|\frac{\hat{x}_{i}}{b}\right|\right)^{-1} \frac{\hat{x}_{i}}{\left|\hat{x}_{i}\right|} .
\end{aligned}
$$

Define parameter $W \triangleq \operatorname{diag}\left[\left(1+\left|\frac{\hat{x}_{i}}{b}\right|\right)\left|\hat{x}_{i}\right|\right]$, then we can simplify (8.b) as follows:

$$
\frac{\partial}{\partial \hat{x}}\left[\sum_{i=1}^{Q} \ln \left(1+\left|\frac{\hat{x}_{i}}{b}\right|\right)\right]=\frac{1}{b} W^{-1} \hat{x} .
$$

To find the extremum of the objective function $J(x)$, we solve the differential equation $\frac{\partial J(x)}{\partial \hat{x}}=0$ and get the following solution of signal $x$ :

$$
\hat{x}=W \Omega^{H} \Lambda y,
$$

where $\Lambda=\left(\lambda I+\Omega W \Omega^{H}\right)^{-1}$, and $\lambda=1 / \beta b$ is a regularization parameter. Since there is cross-dependence between $x$ and $W$, an instinctive thought is to compute these two variables iteratively. The update equations for each iteration are presented here:

$$
(\mathrm{MMP})\left\{\begin{array}{rl}
J(x) & =\ln f_{n}(y-\Omega x)+\ln f(x)=\ln \left[\gamma^{Q} \prod_{i=1}^{Q}\left(1+\left|\frac{x_{i}}{b}\right|\right)^{-2}\right]+\ln \left[\exp \left(-\frac{\|y-\Omega x\|_{2}^{2}}{\sigma_{n}^{2}}\right)\right] \\
& =-2 \gamma^{Q} \sum_{i=1}^{Q} \ln \left(1+\left|\frac{x_{i}}{b}\right|\right)-\frac{\|y-\Omega x\|_{2}^{2}}{\sigma_{n}^{2}} \\
\hat{x}_{\mathrm{MAP}} & =\underset{x}{\arg \min } J(x)=\underset{x}{\arg \min }\left[\sum_{i=1}^{Q} \ln \left(1+\left|\frac{x_{i}}{b}\right|\right)+\beta\|y-\Omega x\|_{2}^{2}\right], \quad \beta=\frac{1}{2 \gamma^{Q} \sigma_{n}^{2}}
\end{array} .\right.
$$




$$
\left\{\begin{array}{l}
\hat{x}^{(k)}=W^{(k-1)}\left(\Omega^{(k-1)}\right)^{H} \Lambda^{(k-1)} y \\
W^{(k)}=\operatorname{diag}\left[\left(1+\left|\frac{\hat{x}_{i}^{(k-1)}}{b_{i}^{(k-1)}}\right|\right)\left|\hat{x}_{i}^{(k-1)}\right|\right] \\
\lambda^{(k)}=2 \gamma^{Q}\left(\sigma_{n}^{(k)}\right)^{2} / b^{(k)} \\
\Lambda^{(k-1)}=\left[\lambda^{(k-1)} I+\left(\Omega^{(k-1)}\right) W^{(k-1)}\left(\Omega^{(k-1)}\right)^{H}\right]^{-1}
\end{array}\right.
$$

where $k$ and $k-1$ in superscript denote the current and previous iteration, respectively. From (11), we can find that besides the variables $x$ and $W$, the regularization parameter $\lambda^{(k)}$ is updated adaptively during the $k$ th iteration, with the update rule using the modified L-curve method in [9]. As the noise level $\sigma_{n}$ will be changed during iterations, we modify the update rule into a function $\lambda=2 \gamma^{Q} \tilde{\sigma}_{n}^{2} / b$, where the noise variance $\tilde{\sigma}_{n}^{2}$ can be computed by $\tilde{\sigma}_{n}^{2}=\|y-\Omega \hat{x}\|_{2}^{2} / L$.

The iterative process will be terminated when the stopping criterion is satisfied:

$$
\Delta J^{(k)}=\left|J\left(\hat{x}^{(k)}\right)-J\left(\hat{x}^{(k-1)}\right)\right| \leq \xi
$$

where $\xi$ represents a prescribed tolerance. When the iteration terminates, the recovered $\hat{x}$ is accepted as the final high-resolution spatial spectrum. Now we can summarize the process of the above algorithm as in Algorithm 1.

\subsection{Analysis of computational complexity}

Due to the similarity of the procedure between the proposed MMP DOA algorithm and the FOCUSS algorithm, the analysis of computational complexity for the MMP DOA algorithm can refer to that of the FOCUSS algorithm. The most time-consuming computation of the whole algorithm in Algorithm 1 is the calculation of the matrix inversion $\Lambda$. With the gradient method, the complexity of this operation is $O\left(Q L^{2}\right)$. By using the conjugate gradient method to accelerate the process, we can reduce the complexity from $O\left(Q L^{2}\right)$ to $O(Q L)$.

\section{Simulation and discussion}

In this section, we exhibit the superior performance of the proposed algorithm from the Monte Carlo statistical analysis as well as the application in the DOA process.

\subsection{Statistical analyses}

In the subsection, we will show the difference in the effectiveness of modeling the sparse signal between the proposed Meridian prior and the Laplace prior. For this purpose, we simulate the reconstruction of signal with different sparse levels. Then, for the same sparsity especially at the higher sparse level, a smaller error of reconstruction means a better model for a sparse signal.

Algorithm 1 MMP DOA algorithm

Task: Estimate DOA $\hat{\vartheta}$ of the target by solving the following optimization problem

$$
\mathrm{MMP} \quad \hat{x}_{\mathrm{MAP}}=\underset{x}{\arg \min } J x=\underset{x}{\arg \min }\left(\sum_{i=1}^{Q} \ln \left(1+\left|\frac{x_{i}}{b}\right|\right)+\beta\|y-\Omega x\|_{2}^{2}\right) .
$$

Data and parameters: Input array data $y$, equal-spaced angle grid $\Theta=\left\{\vartheta_{1}, \ldots, \vartheta_{Q}\right\}$, preconstructed dictionary $\Omega$, the tolerance $\xi, K_{0}$ iterations.

Initialization: Set $k=0$ and $\hat{x}_{0}$ is the $\mathrm{BF}$ result.

Main iteration: Set $k=1$ and do

Weights: Compute $W^{(k)}=\operatorname{diag}\left[\left(1+\left|\frac{\hat{x}_{i}^{(k-1)}}{b_{i}^{(k-1)}}\right|\right)\left|\hat{x}_{i}^{(k-1)}\right|\right]$;

Update: Compute $\hat{x}^{(k)}=W^{(k-1)}\left(\Omega^{(k-1)}\right)^{H} \Lambda^{(k-1)} y$;

Residual: Compute $\Delta J^{(k)}=\left|J\left(\hat{x}^{(k)}\right)-J\left(\hat{x}^{(k-1)}\right)\right|$;

- Return: Set $k=k+1$, if $\Delta J^{(k)}>\xi$ return to "Weights".

Finalize: The angles of the vector $\Theta$ whose index corresponds to the index of the nonzero value of estimated $\hat{x}^{k}$ indicate the estimated DOA of targets. 


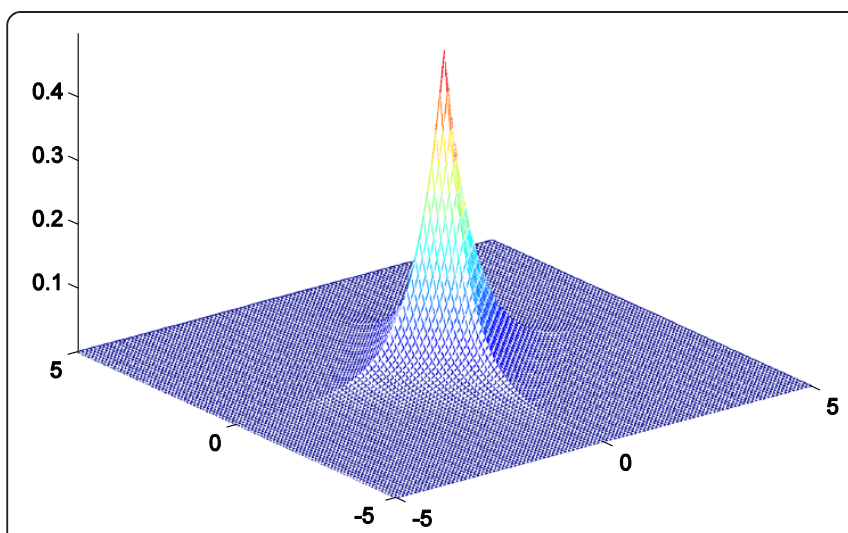

(a)

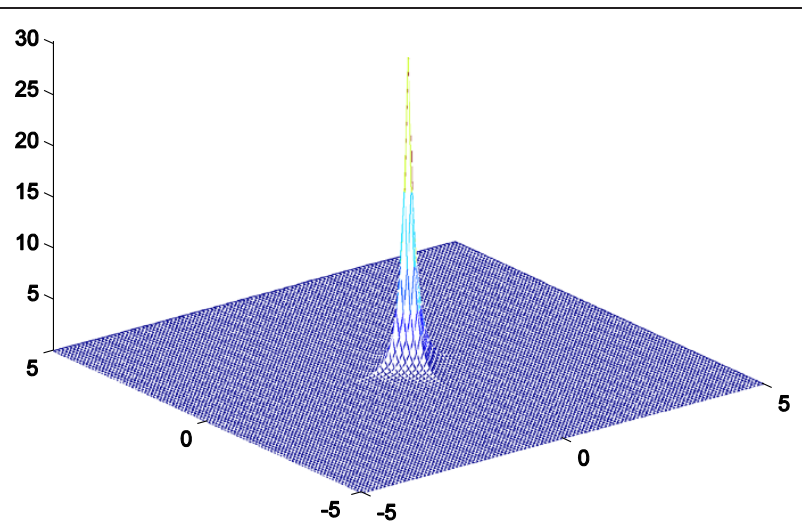

(b)

Figure 1 Distribution of the Laplace and Meridian priors with the same variance. (a) pdf of Laplace distribution. (b) pdf of Meridian distribution.

Without loss of generality, the over-complete dictionary for sparse representation is set to be the matrix of Fourier transform. The over-complete dictionary which is often called as the measurement matrix, is with dimension $N \times M$, where $N=256$ and $M=100$. The testing signal of length $N$, denoted by $v$, is sparse with respect to the Fourier transform, with the number of non-zero entries $K$ ranging from 5 to 40 . The $K$ sparse coefficients satisfy Gaussian distribution $N(0,1)$. Figure 2 depicts the statistical mean squared error (MSE) results under different noise levels using 100 Monte Carlo experiments.

From Figure 2, we can find that the MSE results of the MMP algorithm are much smaller than those of the algorithm based on the Laplace prior, for both high noise level (signal-to-noise ratio $(\mathrm{SNR})=40 \mathrm{~dB}$ ) or low noise level
$(\mathrm{SNR}=10 \mathrm{~dB})$. Especially, in Figure 2a, even at the smallest sparse level $(K=5)$, the MSE of the algorithm based on the Laplace prior is already at the order of $10^{-1}$, which is about ten times bigger than that of the MMP algorithm at the highest sparse level $(K=40)$, not to mention if it is compared with that of the MMP algorithm at the same sparse level $(K=5)$. The same trend is also presented in Figure $2 \mathrm{~b}$. Thus, we can give the conclusion that the Meridian prior is a better model than the Laplace model for sparse signal.

\subsection{Application in DOA estimation}

In this subsection, the proposed MMP algorithm is applied for both linear and planar arrays. We first consider a uniform linear array (ULA) of 15 sensors equally spaced by a half wavelength and the carrier frequency of

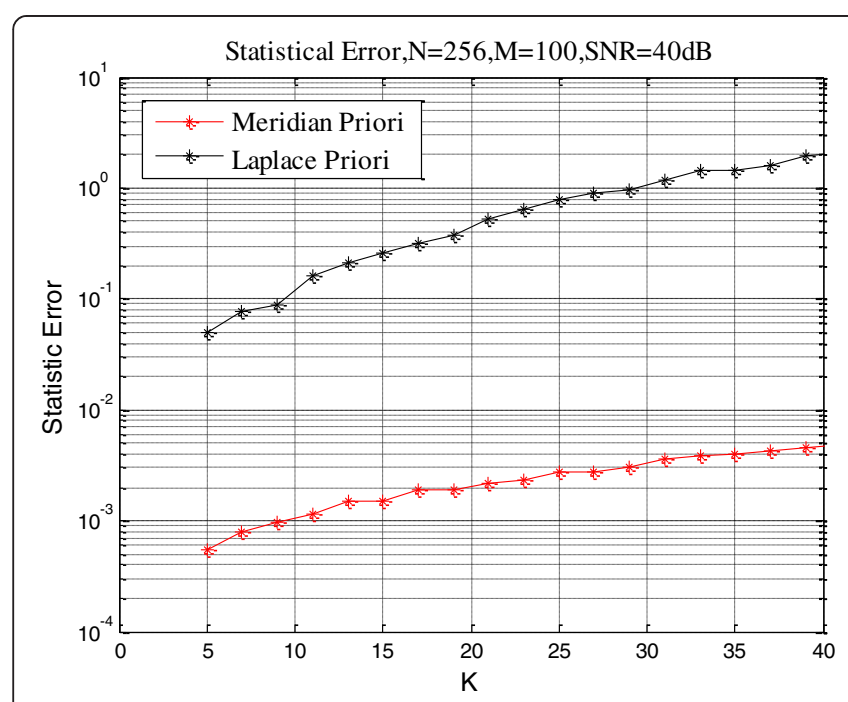

(a)

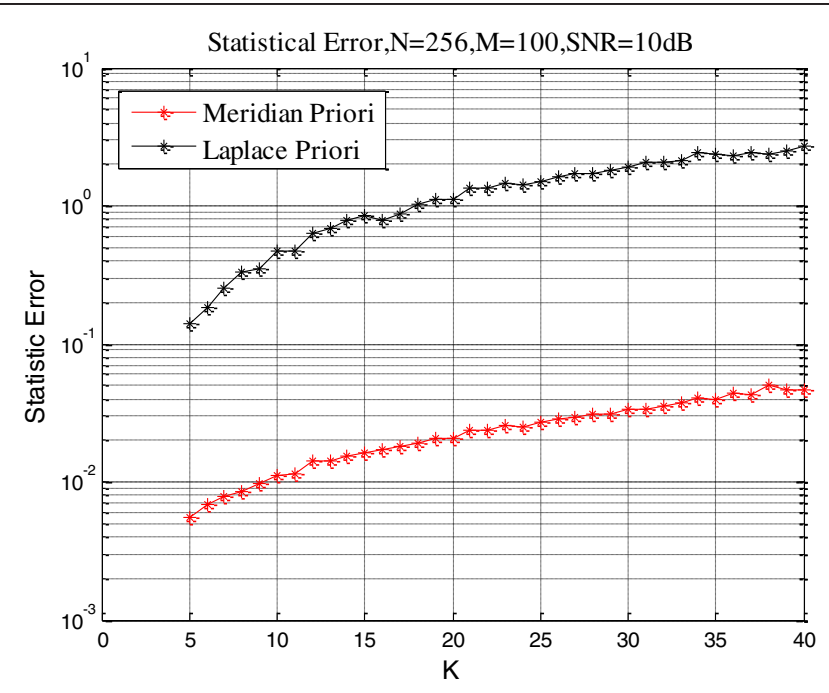

(b)

Figure 2 Statistic errors with different support sizes $K$ and SNRs. (a) Statistic comparison under high SNR and (b) statistic comparison under low SNR. 


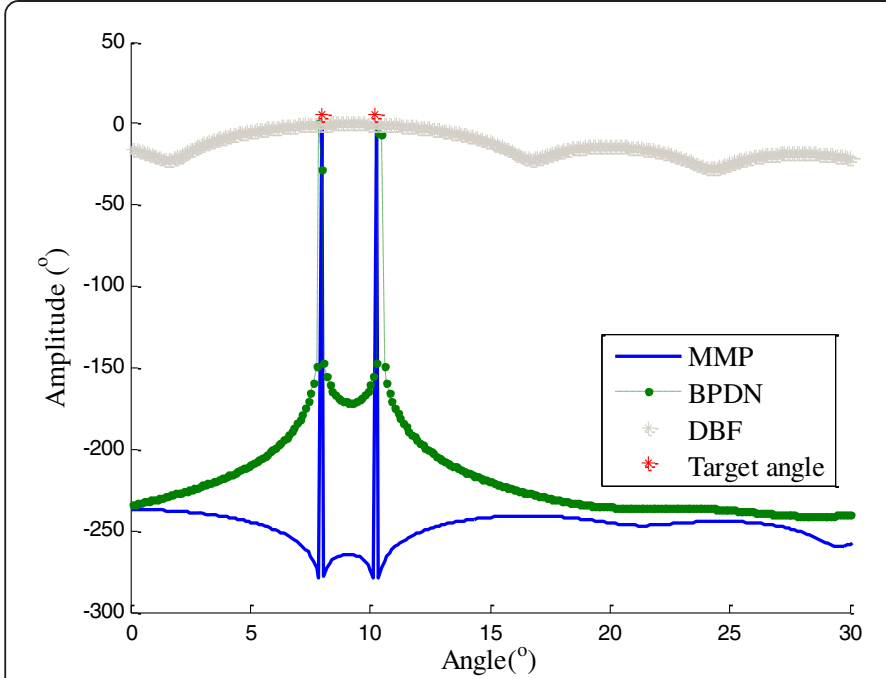

(a)

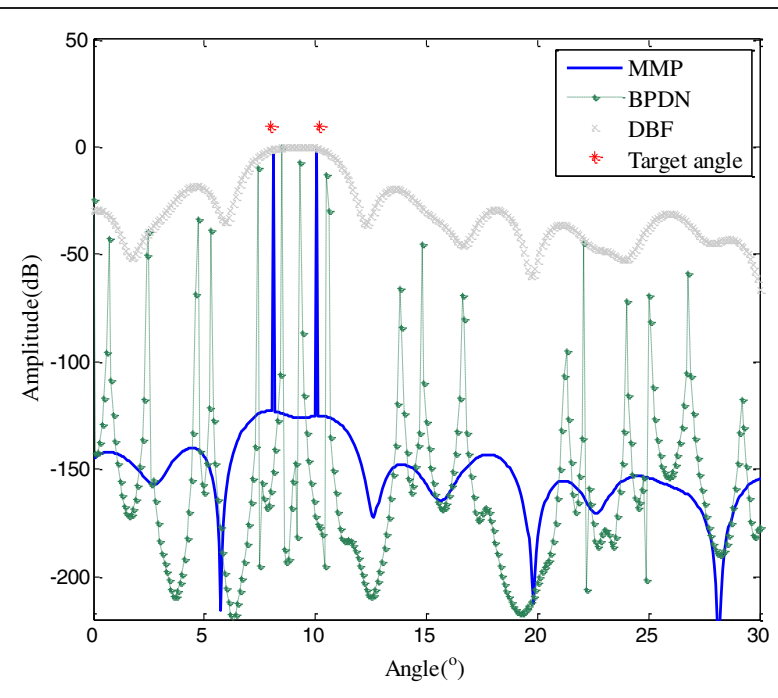

(b)

Figure 3 Results of one-dimensional DOA estimation. (a) $S N R=40 \mathrm{~dB}$. (b) $\mathrm{SNR}=5 \mathrm{~dB}$.

the transmitted signal being $300 \mathrm{MHz}$. From the far field, two coherent target echoes contaminated with Gaussian noise impinge on the array. The horizontal distances of the two targets from the center of the sensors are both $100 \mathrm{~km}$. Two targets are placed at angles $8^{\circ}$ and $10.2^{\circ}$, respectively. Only one snapshot is considered to illustrate the performance of the proposed method in DOA estimation. In order to test the robustness of noise, the simulations are implemented under different noise levels. Figure 3 shows the reconstructed spectrum of the proposed MMP algorithm compared with that of digital $\mathrm{BF}(\mathrm{DBF})$ and BPDN.

As indicated in Figure 3a, even though the noise energy is small $(\mathrm{SNR}=40 \mathrm{~dB})$, the DBF method cannot distinguish the two targets, whereas BPDN and the proposed MMP are both capable to do that. In Figure 3b, in the heavily noisy environment ( $\mathrm{SNR}=5 \mathrm{~dB}$ ), the two targets cannot be identified by the BPDN method since many false peaks appear in the reconstructed spatial spectrum, while they still can be separated by the MMP algorithm.

Next, we extend the DOA estimation from ULA to a uniform rectangular array (URA). For simplicity, we consider a URA consisting of $16 \times 16$ identical isotropous sensors. The intervals of sensor array in the horizontal and vertical direction are both $1.5 \lambda$, where $\lambda$ is the center wavelength, and the carrier frequency is $300 \mathrm{MHz}$. We assume narrowband coherent signals reflected by two pairs of close targets which impinge on the URA from different directions that are indicated by the azimuth and elevation of the target signal, which are $\left(-5.1^{\circ},-7.81^{\circ}\right),\left(-5.8^{\circ},-9^{\circ}\right)$, $\left(4.98^{\circ}, 9^{\circ}\right)$, and $\left(5^{\circ}, 7.91^{\circ}\right)$, respectively. SNR is fixed as $5 \mathrm{~dB}$, and one snapshot is available.

Figure 4 shows the intensity map of the DBF, BPDN, and MMP, which is a function of azimuth and elevation. As shown in Figure 4a, DBF fails to separate two pairs of highly close targets, and each of the two close targets are

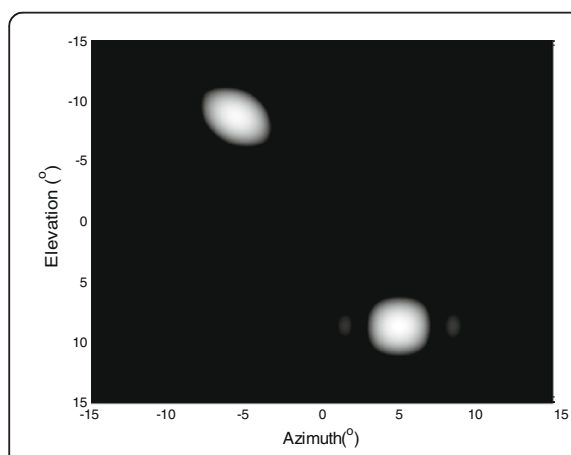

(a)

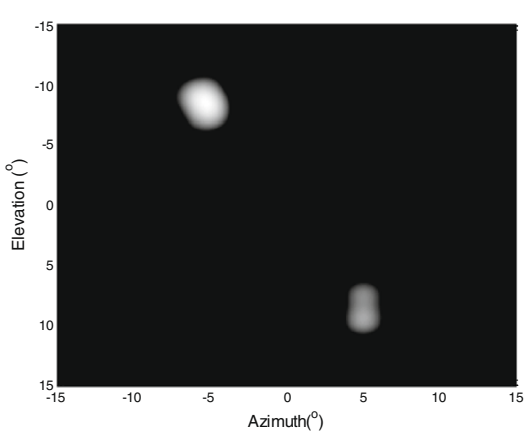

(b)

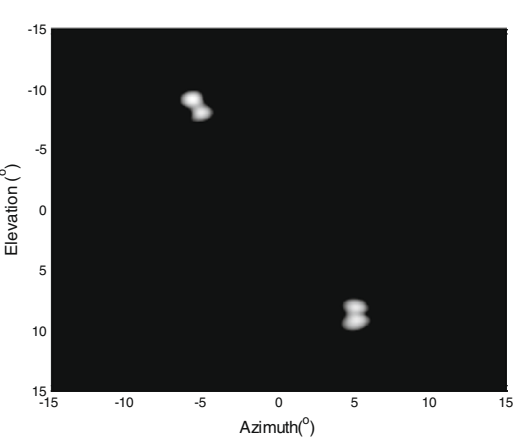

(c)

Figure 4 Results of two-dimensional DOA estimation. (a) DBF, (b) BPDN, and (c) MMP. 


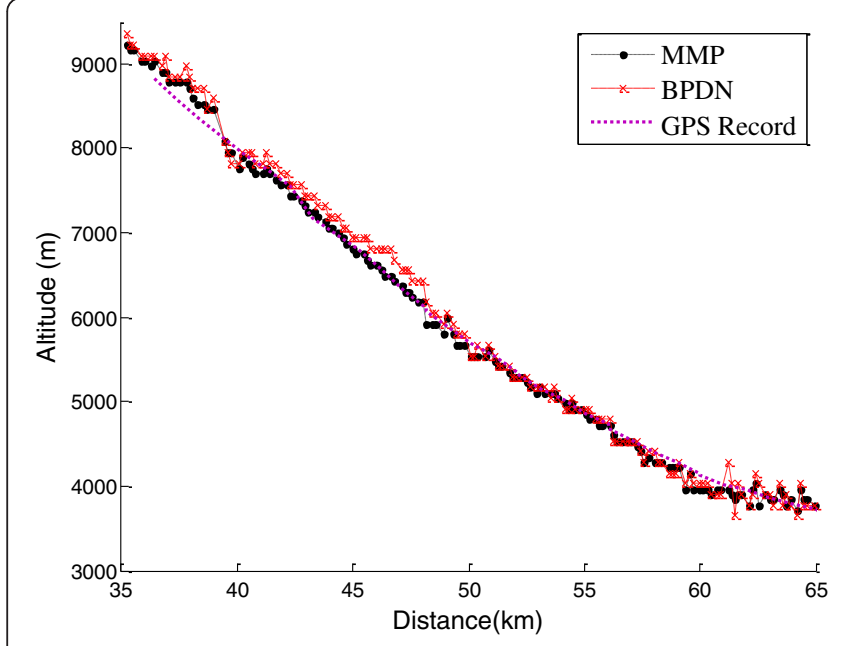

(a)

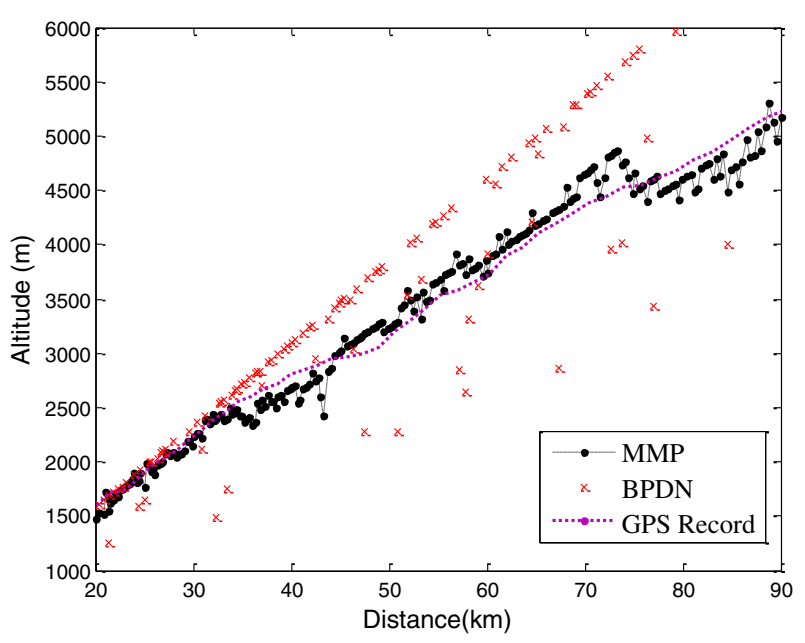

(b)

Figure 5 Estimation results of ground truth data of VHF radar. (a) Altitude estimation of a landing plane, and (b) altitude estimation of a plane taking off.

merged into one. The range of each pair of blurred targets resulting from the BPDN algorithm is smaller than that of the DBF approach, whereas each pair of close targets still cannot be distinguished. In Figure 4c, four targets are explicitly identified via the proposed MMP algorithm.

Based on the above analysis, we believe that the proposed MMP algorithm achieves better performance than the conventional matching-based spectrum algorithm and popular sparse-based algorithms even when the available data are severely insufficient. Especially, when the SNR is low, i.e., the contaminated echoes include more noise energy, since the sparsity of the signal can be enhanced by Meridian prior, the target energy within the spatial spectrum via the MMP algorithm can be greater than that of other algorithms.

\subsection{Process of the ground truth data from VHF radar}

In this subsection, we use the ground truth data of the very high frequency (VHF) radar located on a flat ground to verify the effectiveness of the proposed MMP algorithm. Since the VHF radar is configured with a wide beam, the received echo is the mixture of the direct echoes and the specular components resulting from multipath effects. The multipath components are coherent with the direct echoes, which increases the difficulty in estimating the DOA of targets.

A planar array including double-parallel linear arrays is placed on the VHF radar. Each sub-array consists of eight sensors with $1.78 \lambda$ spacing in the vertical direction, and each sensor in this planar array is horizontally polarized. The distance between the double-parallel linear arrays is $1.12 \mathrm{~m}$. With the distance between the array and the moving target, we can obtain the altitude of the target via the triangle geometry relationship. Figure 5a presents the altitude estimation results of data received when the plane is landing, where the radar is set beside a lake. Figure $5 \mathrm{~b}$ shows the altitude estimation results when the plane is taking off, where the radar is set in an airport.

Due to the different settings of the recording environment, the noise components as well as the diffuse components are much less in the first received data. So both the BPDN and the MMP algorithm can achieve satisfactory estimations, as shown in Figure 5a. However, in Figure 5b, due to the effect of the noise and random diffuse components, the sparsity property of the spatial spectrum is destroyed; in other words, the current spatial spectrum is no longer sparse, but with many false peaks. Since the classical Laplace prior-based BPDN algorithm can only work under the sparse assumption, it cannot work well under this circumstance. On the other hand, because the Meridian distribution has the characteristic of high energy concentration, it can suppress the small elements (such as noise and diffuse components) during the process and finally generates a sparse spectrum. So, our proposed MMP algorithm can achieve better performance.

\section{Conclusions}

In this paper, considering that the Meridian distribution has the characteristic of high energy concentration, we proposed to adopt the Meridian prior as the prior distribution of the coefficient vector when a problem of sparse representation is to be solved. Further, we developed a new Meridian prior-based algorithm, i.e., the MMP DOA algorithm, to achieve high-resolution DOA estimation. Since the Meridian prior imposes a more stringent 
constraint on the sparsity of signal, the proposed MMP algorithm is superior to the conventional method that minimizes the $l_{1}$-norm of signal, equivalent to imposing a Laplace prior on a signal. Both the results of simulations and the results of ground truth data process prove a good performance of the proposed MMP algorithm in high-resolution DOA estimation.

\section{Competing interests}

The authors declare that they have no competing interests.

\section{Acknowledgment}

This work is supported in part by the National Science Foundation of China under grants 60902079, 61201289, 61033004, 61072104, and 61070138 and by the Fundamental Research Funds for the Central Universities K50510020018

\section{Author details}

${ }^{1}$ School of Electronic Engineering, Xidian University, Xi'an 710071, China.

${ }^{2}$ No.8511 Research Institute of CASIC, Nanjing 210007, China.

Received: 30 June 2012 Accepted: 21 February 2013

Published: 29 April 2013

\section{References}

1. RO Schmidt, Multiple emitter location and signal parameter estimation. IEEE Trans. Antenn. Propag. 43(3), 276-280 (1986)

2. R Roy, T Kailath, ESPRIT-estimation of signal parameters via rotational invariance techniques. IEEE Trans. Acoust. Speech Signal Process 37(7), 984-995 (1989)

3. DH Johnson, DE Dudgeon, Array Signal Processing: Concepts and Techniques (Upper Saddle River, Prentice Hall, 1993)

4. TJ Shan, M Wax, T Kailath, On spatial smoothing for estimation of coherent signals. IEEE Trans. Acoust. Speech Signal Process 33(no.4), 806-811 (1985)

5. H Krim, M Viberg, Two decades of array signal processing research: the parametric approach. IEEE Signal Process 13(4), 67-94 (1996)

6. M Viberg, B Ottersten, Sensor array processing based on subspace fitting. IEEE Trans. Signal Process 39(5), 1110-1121 (1991)

7. IF Gorodnitsky, BD Rao, Sparse signal reconstructions from limited data using FOCUSS: a re-weighted minimum norm algorithm. IEEE Trans. Signal Process 45(3), 600-616 (1997)

8. D Malioutov, M Cetin, A Willsky, A sparse signal reconstruction perspective for source localization with sensor arrays. IEEE Trans. Signal Process 53(8), 3010-3022 (2005)

9. BD Rao, K Engan, SF Cotter, J Palmer, KK Delgado, Subset selection in noise based on diversity measure minimization. IEEE Trans. Signal Process 51(3), 760-770 (2003)

10. MM Hyder, K Mahata, Direction-of-arrival estimation using a mixed $I_{2,0}$ norm approximation. IEEE Trans. Signal Process 58(9), 4646-4655 (2010)

11. RE Carrillo, KE Barner, TC Aysal, Robust sampling and reconstruction methods for sparse signals in the presence of impulsive noise. IEEE J. Sel. Top Signal Process 4(2), 392-408 (2010)

12. AC Gurbuz, JH McClellan, A compressive beamforming method, in Proceedings of IEEE International Conference on Acoustics, Speech and Signal Processing (ICASSP'08) (Las Vegas, IEEE, 2008). pp. 2617-2620

13. D Donoho, Compressed sensing. IEEE Trans. Inf. Theory 52(4), 5406-5425 (2006)

14. EJ Candès, J Romberg, T Tao, Robust uncertainty principles. Exact signal reconstruction from highly incomplete frequency information. IEEE Trans. Inf. Theory 52(2), 489-509 (2006)

15. S Chen, D Donoho, M Saunders, Atomic decomposition by basis pursuit. SIAM J. Sci. Comput. 20(1), 33-61 (1998)

16. R Tibshirani, Regression shrinkage and selection via the lasso. J. Roy. Stat. Soc. B 58(1), 267-288 (1996)

17. MAT Figueiredo, RD Nowak, SJ Wright, Gradient projection for sparse reconstruction: application to compressed sensing and other inverse problems. IEEE J. Sel. Top Signal Process 1(4), 586-597 (2007)

18. E Candes, M Wakin, S Boyd, Enhancing sparsity by reweighted $I_{1}$ minimization. J. Fourier Anal. Appl. 14(5), 877-905 (2008)
19. J Jrzasko, A Manduca, Highly undersampled magnetic resonance image reconstruction via homotopic /o minimization. IEEE Trans. Med. Imaging 28(1), 106-121 (2009)

20. SD Babacan, R Molina, AK Katsaggelos, Bayesian compressive sensing using Laplace priors. IEEE Trans. Image Process 19(1), 53-63 (2010)

21. R Chartrand, Exact reconstruction of sparse signals via nonconvex minimization. IEEE Signal Process Lett. 14(10), 707-710 (2007)

doi:10.1186/1687-6180-2013-91

Cite this article as: Zhao et al.: High-resolution DOA estimation with

Meridian prior. EURASIP Journal on Advances in Signal Processing 2013 2013:91.

\section{Submit your manuscript to a SpringerOpen ${ }^{\odot}$ journal and benefit from:}

- Convenient online submission

- Rigorous peer review

- Immediate publication on acceptance

- Open access: articles freely available online

- High visibility within the field

- Retaining the copyright to your article

Submit your next manuscript at $>$ springeropen.com 